\title{
Coherent terahertz control of antiferromagnetic spin waves
}

\author{
Tobias Kampfrath ${ }^{1,2,3 * \star}$, Alexander Sell ${ }^{1 *}$, Gregor Klatt', Alexej Pashkin', Sebastian Mährlein',

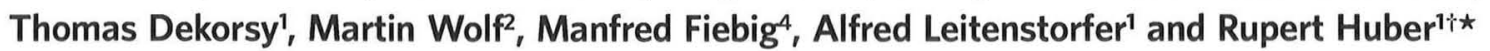

\begin{abstract}
Ultrafast charge and spin excitations in the elusive terahertz regime $^{1,2}$ of the electromagnetic spectrum play a pivotal role in condensed matter ${ }^{3-13}$. The electric field of free-space terahertz pulses has provided a direct gateway to manipulating the motion of charges on the femtosecond timescale ${ }^{6-9}$. Here, we complement this process by showing that the magnetic component of intense terahertz transients enables ultrafast control of the spin degree of freedom. Single-cycle terahertz pulses switch on and off coherent spin waves in antiferromagnetic NiO at frequencies as high as $1 \mathrm{THz}$. An optical probe pulse with a duration of $\mathbf{8}$ fs follows the terahertz-induced magnetic dynamics directly in the time domain and verifies that the terahertz field addresses spins selectively by means of the Zeeman interaction. This concept provides a universal ultrafast means to control previously inaccessible magnetic excitations in the electronic ground state.

The latest advances in terahertz technology $y^{1,2}$ have made it possible to observe $\mathrm{e}^{3-5}$ and even control ${ }^{6-9}$ low-energy elementary excitations in solids (such as lattice vibrations, plasma oscillations or quasiparticles in superconductors) on the femtosecond timescale. In all these experiments, the terahertz field manipulates the motion of charge carriers by electric-dipole coupling. Interaction with magnetic dipoles is usually much weaker and has been harnessed only recently in artificial metamaterials ${ }^{10,11}$. However, magnetic terahertz interaction also holds the potential for ultrafast spin control, as the spin $\mathbf{S}$ of an electron is associated with a magnetic moment $\gamma \mathcal{S}$, where $\gamma$ denotes the gyromagnetic constant. The most direct mode of spin manipulation is therefore provided by a time-dependent magnetic field $\mathbf{B}$, which exerts a Zeeman torque given by
\end{abstract}

$$
\mathbf{G}=\gamma \mathbf{S} \times \mathbf{B}
$$

In antiferromagnets, which represent the largest class of spinordered materials in nature, collective spin precessions, so-called magnons, typically occur at frequencies as high as $1 \mathrm{THz}$ (ref. 12). Despite their significance in strongly correlated materials ${ }^{13}$, achieving time-resolved access to these quasiparticles has been challenging. Zeeman-type manipulation ${ }^{14-16}$ has not been possible with antiferromagnets due to a lack of sufficiently fast magnets. Indirect coupling via stimulated Raman scattering has been limited to special orthoferrites with giant spin-orbit coupling ${ }^{17}$. Optical pulses heating the electron system have manipulated the equilibrium spin direction ${ }^{18-21}$, but the associated cooling dynamics limits the speed.
Here, we explore the interplay of an antiferromagnet with highly intense terahertz pulses ${ }^{22,23}$, with a time resolution of $8 \mathrm{fs}$. Surprisingly, the large terahertz electric field of up to $0.4 \mathrm{MV} \mathrm{cm}^{-1}$ is found to make no measurable contribution to the observed dynamics. On the other hand, the magnetic component couples to electron spins selectively by the Zeeman interaction, and allows for a novel universal scheme of femtosecond spin control in the orbital ground state.

The prototypic antiferromagnet $\mathrm{NiO}$ is used to demonstrate the feasibility of our idea. Below the Neel temperature of $523 \mathrm{~K}$, the $\mathrm{Ni}^{2+}$ spins are aligned in parallel within $\{111\}$ planes (Fig. 1a), where they point along the $\langle 11 \overline{2}\rangle$ axes $^{24}$. Adjacent planes are oppositely magnetized due to antiferromagnetic coupling. Each of the four equivalent $\langle 111\rangle$ stacking directions defines a twin (T) domain that is subdivided into spin (S) domains ${ }^{24,25}$. Birefringence induced by a slight lattice contraction along the stacking axes allows us to map the domain structure with a polarizationsensitive microscope (Fig. 1b). For our free-standing $\mathrm{NiO}$ single crystal (thickness, $d=45 \mu \mathrm{m}$ ), we find dimensions of the $\mathrm{T}$ domains of the order of $100 \mu \mathrm{m}$. The $\mathrm{S}$ domains are not resolved, as they are less than $1 \mu \mathrm{m}$ wide ${ }^{25}$. Inelastic neutron and light scattering as well as infrared absorption have identified an antiferromagnetic magnon mode in $\mathrm{NiO}$ at $1 \mathrm{THz}$ (refs 24,26).

Figure $1 \mathrm{c}$ presents a schematic of our femtosecond experiment. A highly intense terahertz transient is focused onto a single T domain (white circle, Fig. 1b). Figure 2a shows the time trace $B(t)$ of the incident magnetic pulse. This single-cycle transient reaches a peak magnetic field of $0.13 \mathrm{~T}$, combined with an electric field of as much as $0.4 \mathrm{MV} \mathrm{cm}^{-1}$, and covers a broad spectral range from 0.1 to $3 \mathrm{THz}$, fully overlapping the magnon resonance at $1 \mathrm{THz}$. An $8-\mathrm{fs}$ pulse (central photon energy, $1 \mathrm{eV}$ ) probes the terahertz-driven dynamics at a variable delay time $t$. As it is not clear a priori how the terahertz fields interact with the sample, we analyse both the polarization state and the intensity of the transmitted probe. In particular, an induced magnetization $\mathbf{M}(t)$ manifests itself by the Faraday effect, where the projection of $\mathbf{M}(t)$ onto the propagation direction $e_{k}$ of the probe pulse causes a transient circular birefringence that rotates the linear probe polarization by an angle

$$
\theta_{\mathrm{F}}(t)=V d\left\langle\mathbf{e}_{\mathbf{k}} \cdot \mathbf{M}(t)\right\rangle
$$

Here, $V$ is the magneto-optic Verdet constant, and the angular brackets denote averaging over the relevant sample volume. In fact, we find that the terahertz transient exclusively causes a Faraday rotation $\theta_{\mathrm{F}}$ of the probe polarization. A possible pumpinduced probe ellipticity is found to be below the noise floor of

'Department of Physics and Center for Applied Photonics, University of Konstanz, Universitätsstraße 10, 78464 Konstanz, Germany, ${ }^{2}$ Fritz-Haber-Institut der Max-Planck-Gesellschaft, Faradayweg 4-6, 14195 Berlin, Germany, ${ }^{3}$ FOM Institute for Atomic and Molecular Physics (AMOLF), Science Park 104, 1098 XG Amsterdam, The Netherlands, ${ }^{4}$ Helmholtz-Institut für Strahlen- und Kernphysik, Nußallee 14-16, 53115 Bonn, Germany; ${ }^{\dagger}$ Present address: Department of

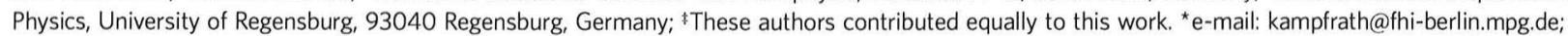
rupert.huber@uni-konstanz.de 

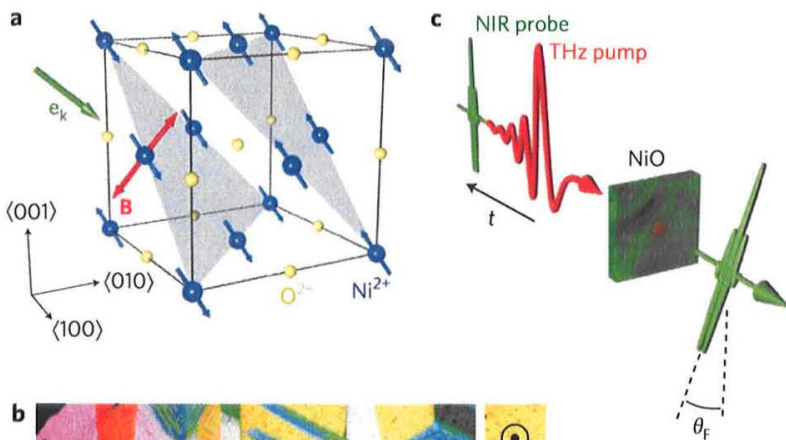

b

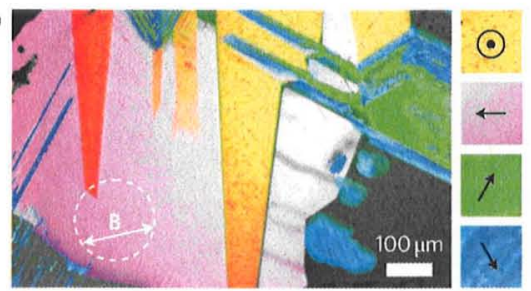

Figure 1 | The experiment. a, Crystal lattice of $\mathrm{NiO}$ (blue spheres, $\mathrm{Ni}^{2+}$; yellow spheres, $\mathrm{O}^{2-}$ ) with magnetically ordered spins (blue arrows) of a selected $\mathrm{S}$ domain in the (111) planes (light blue) and the direction of the terahertz magnetic field $\mathbf{B}$ (double-ended red arrow). b, Polarization micrograph (top view) of our $45-\mu \mathrm{m}$-thick $\mathrm{NiO}$ sample, identifying all four types of T domains. The projection of the stacking axis of the spin planes is indicated by the black arrows in the key. The specimen was cut from a single crystal, grown by the flame fusion method, and annealed in oxygen at a temperature of $1,400 \mathrm{~K}$. The white broken circle and the double-ended arrow illustrate the area of the terahertz excitation spot and the polarization of the magnetic field, respectively. The experimental geometry of the terahertz field with respect to the spin structure is depicted in a. c, Schematic of femtosecond terahertz spin resonance. An intense free-space terahertz transient (red curve, magnetic field component) interacts with the electron spins of a sample to launch a coherent magnon wave. A femtosecond near-infrared (NIR) probe pulse (green curves) co-propagating along the direction $\mathbf{e}_{\mathbf{k}}$ samples the induced net magnetization by means of the Faraday effect, after a variable delay time $t$.

$\sim 5 \times 10^{-6}$, showing that effects like field-driven linear birefringence are much less important than $\theta_{\mathrm{F}}$. Similarly, we do not resolve any measurable terahertz-induced transmittance changes within the large bandwidth of our probe. The absence of a spontaneous magnetization in an antiferromagnet renders the detection of Faraday rotation much more challenging than in conventional ferromagnets. It is the unique stability of our hybrid laser system (see Methods) that allows us to monitor the spin dynamics, nevertheless, with high sensitivity.

The time trace of the Faraday rotation $\theta_{\mathrm{F}}$ is displayed in Fig. $2 \mathrm{~b}$. A harmonic oscillation with a period of 1 ps sets in within a single cycle, reaches its maximum amplitude at $t=3 \mathrm{ps}$, and decays exponentially with a time constant of 29 ps thereafter. The Fourier transform of the Faraday transient consists of a narrow peak at $1.0 \mathrm{THz}$ (Fig. 2, inset), clearly linking the signal to the high-frequency spin eigenmode in $\mathrm{NiO}$. The traces show no indications of excess heating in the form of a slowly varying signal background, as is usually observed in spin-manipulation schemes using optical pump pulses ${ }^{17}$. Freecarrier and lattice absorption are negligible, because $\mathrm{NiO}$ is an insulator and all optical phonon resonances are located above $12 \mathrm{THz}$, far beyond the frequencies considered here. Most remarkably, we find that the observed dynamics are solely driven by the magnetic, not the electric field. Because the Faraday signal is proportional to the pump field (Supplementary Fig. S1), electricdipole interaction with magnons would represent a linear magneto-electric effect. In centrosymmetric materials such as $\mathrm{NiO}$, such processes are forbidden ${ }^{27}$.

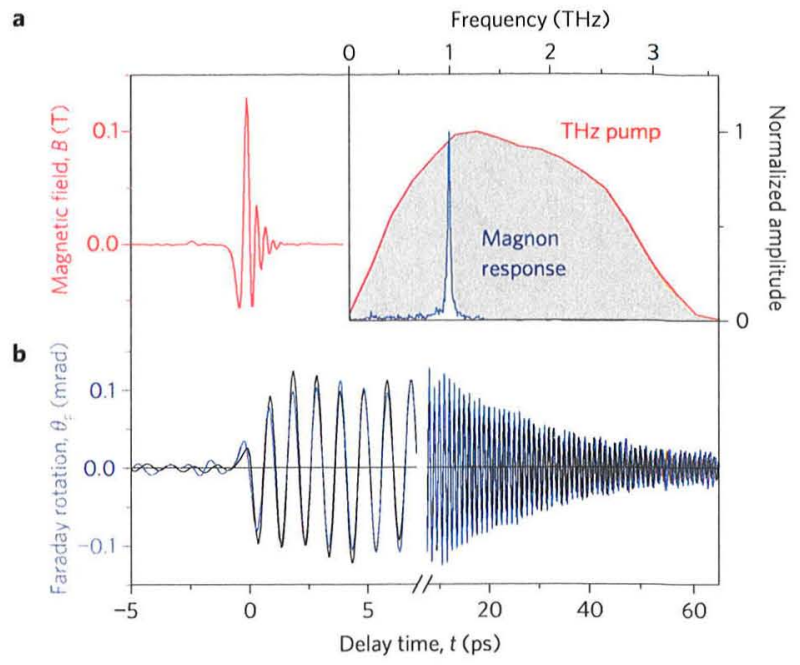

Figure 2 | Femtosecond terahertz spin resonance. a, Magnetic field of the incident terahertz pulse as a function of time $t$. $\mathbf{b}$, Ultrafast Faraday rotation $\theta_{\mathrm{F}}(t)$ induced in the $\mathrm{NiO}$ sample (see Fig. 1) at room temperature, detected by an $8 \mathrm{fs}$ probe pulse. Harmonic oscillations with a period of 1 ps are due to an antiferromagnetic spin precession. Blue curve, experiment; black line, simulation based on the Hamiltonian of equation (3) with a Gilbert constant of $2.1 \times 10^{-4}$ (amplitude scaled by a factor of 0.7 ). Inset: amplitude spectra of the Faraday transient in $\mathbf{b}$ and the driving terahertz field in $\mathbf{a}$.

To gain further insight into the details of the spin motion and its driving force, we model the magnon dynamics microscopically. The relevant excitation mode is adequately described as a uniform precession of all spins located on the two antiferromagnetic sublattices. We adapt the Hamiltonian of ref. 26 to read

$$
H=-J \mathbf{S}_{1} \cdot \mathbf{S}_{2}+\sum_{i=1}^{2}\left[D_{x} S_{i x}^{2}+D_{y} S_{i y}^{2}\right]+\gamma \mathbf{B}(t) \cdot \sum_{i=1}^{2} \mathbf{S}_{i}
$$

The first term describes the exchange interaction between adjacent electron spins $\mathbf{S}_{1}$ and $\mathbf{S}_{2}$ in the two sublattices, where $J$ is negative and accounts for antiferromagnetic coupling. The second term captures intrinsic anisotropy effects owing to spin-spin dipole interaction and the influence of the crystal potential mediated by spinorbit coupling. These contributions ensure a preferential orientation along the $\langle 11 \overline{2}\rangle$ direction in the $\{111\}$ easy plane. Finally, the last term describes the Zeeman energy in the external field $\mathbf{B}(t)$. We derive a Landau-Lifshitz-Gilbert equation of motion, solve numerically for the net magnetization $\mathbf{M} \propto \mathbf{S}_{1}+\mathbf{S}_{2}$, and compute the resulting Faraday rotation using equation (2). For each $\mathrm{T}$ domain, an average over the three possible $\mathrm{S}$ domains is performed. All parameters of equation(3) have been determined previously, for instance by neutron scattering experiments ${ }^{24,26}$. We take the actual measured driving fields (Fig. 2a) and account for multiple reflections at the surfaces of the $\mathrm{NiO}$ crystal. The Gilbert damping constant is adjusted to $(2.1 \pm 0.1) \times 10^{-4}$, which best replicates the measurement.

The simulation reproduces well the temporal shape, phase and amplitude of the experimental signal (Fig. 2b). Our model therefore quantitatively confirms the magnetic nature of the terahertzmagnon coupling, and allows a slow-motion picture of the microscopic dynamics to be prepared (see Supplementary Information). The Zeeman torque turns the spins out of the easy plane, and the anisotropy field forces them onto a precessional motion about their equilibrium direction. The out-of-plane spin component is 

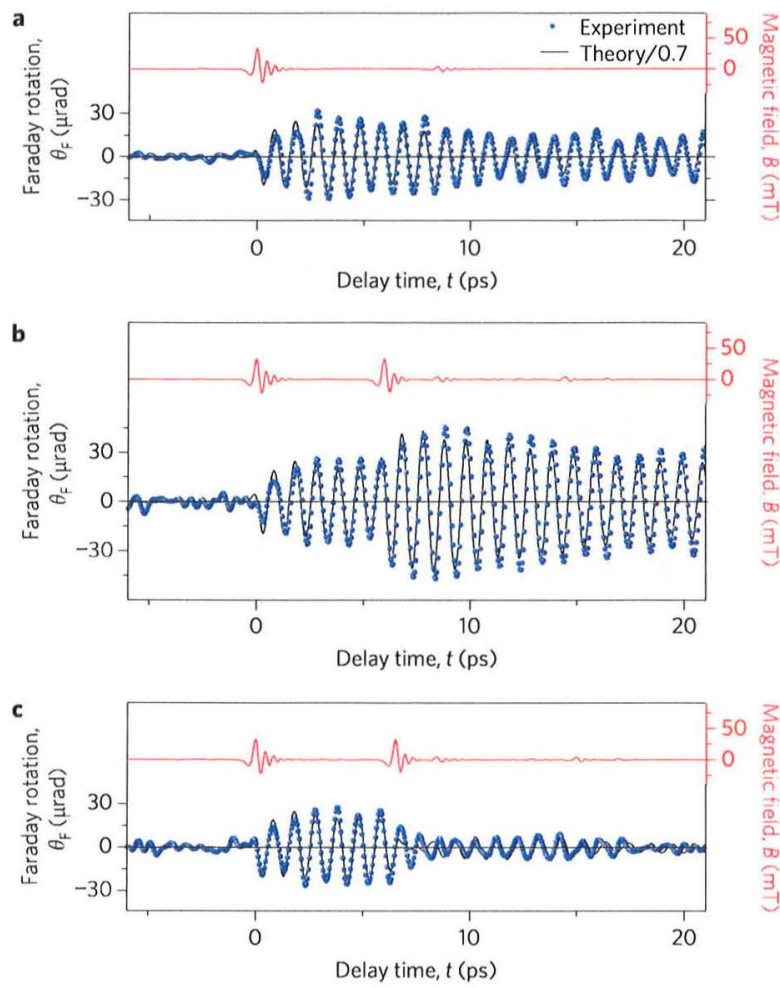

Figure 3 | Coherent terahertz control of spin waves. a, Faraday rotation (dots, experiment; line, numerical simulation) induced by a single terahertz magnetic field pulse (red curve). b, Excitation by two pulses (red curve) with a mutual time delay of $\Delta t=6 \mathrm{ps}$ launches a coherent magnon oscillation and subsequently, enhances its amplitude by a factor of almost 2 (shaded area). c, Excitation with $\Delta t=6.5 \mathrm{ps}$ switches the magnon on and off coherently.

exactly antiparallel in the two sublattices, whereas the in-plane coordinate oscillates in the same direction, adding to a measurable dynamic magnetization. For the transient of Fig. 2a, we find that the spins are deflected from their equilibrium direction by up to $0.4^{\circ}$. Following the ultrashort driving field, the magnetization decays mono-exponentially, suggesting that the relaxation is dominated by a single process such as magnon-magnon or magnonphonon scattering ${ }^{28}$.

The high degree of coherence of the observed dynamics benefits from the fact that the terahertz pulse exclusively addresses the electron spins and does not deposit excess heat in other degrees of freedom. Even after complete relaxation of the magnon wave, we estimate that the energy deposited by the terahertz pump pulse increases the temperature of the excited $\mathrm{NiO}$ volume by less than $10 \mu \mathrm{K}$. Terahertz magnetic fields, therefore, qualify uniquely for ultrafast coherent control experiments. To demonstrate the potential of this idea, we now excite $\mathrm{NiO}$ by three different pulse sequences as shown in Fig. 3. First, a single terahertz transient triggers an exponentially decaying spin precession as discussed above (Fig. 3a). Next, by introducing a second identical pulse after a delay of $\Delta t=6 \mathrm{ps}$, we double the amplitude of the induced magnetization (Fig. 3b). Here, the second transient induces a torque that is in phase with the precession caused by the first pulse. As the dephasing time greatly exceeds the interval $\Delta t$ between the two pulses, this process is excellently coherent. Finally, the enhancement effect may be reversed if the second pulse arrives 6.5 precession cycles after the first one, that is, if $\Delta t=6.5 \mathrm{ps}$ (Fig. 3c). The torque by the second pulse is now out of phase with the spin precession and effectively switches off the dynamics. As with nuclear magnetic resonance experiments, our scheme lends itself to arbitrary pulse sequences and tailored wave forms. Such femtosecond terahertz spin resonance may extend exciting approaches such as spin-echo experiment $^{29}$ to antiferromagnets and ultrashort timescales. Our magneto-optical detection scheme offers the additional benefit of probing the spatial structure of the induced magnetization with micrometre resolution, opening promising applications for magnetic nanostructures ${ }^{14}$.

In summary, we show that intense single-cycle terahertz transients allow for femtosecond coherent control of previously inaccessible antiferromagnetic magnons, by means of pure magnetic-field coupling. Most importantly, the resonant terahertz driving force leaves other degrees of freedom unexcited and, thus, reveals ultrafast spin dynamics in the orbital ground state. Coherent magnons may now be used as probes for ultrafast interactions of the electron spin with the orbital motion and lattice modes in essentially all terahertz-transparent matter and at all relevant frequencies. This principle is expected to be widely scalable. Our simulations anticipate a novel regime of dramatic terahertz nonlinearities beyond the perturbative regime once the driving field approaches amplitudes of $10 \mathrm{~T}$. Such values have indeed been achieved recently in the mid-infrared ${ }^{30}$ and may soon also become available at frequencies below $10 \mathrm{THz}$.

\section{Methods}

Experimental setup. The experimental setup is based upon a hybrid laser system combining a femtosecond multi-branch erbium:fiber unit with a high-power Ti:sapphire amplifier ${ }^{30}$. The table-top facility provides $8 \mathrm{fs}$ near-infrared pulse (pulse energy, $0.5 \mathrm{~nJ}$; photon energy, $1 \mathrm{eV}$ ) synchronized with $5 \mathrm{~mJ}$ pulses with a duration of $100 \mathrm{fs}$ (photon energy, $1.55 \mathrm{eV}$; repetition rate, $1 \mathrm{kHz}$ ). The latter output is optically rectified in a large-area $\langle 110\rangle$-oriented ZnTe crystal with a thickness of $0.5 \mathrm{~mm}$ to generate intense free-space terahertz pump transients. The terahertz magnetic and electric fields are related by $B(t)=E(t) / c$, where $c$ represents the speed of light in vacuum. $E(t)$ is directly recorded by electro-optic sampling in a $\langle 110\rangle$-oriented GaP crystal. These transients are focused onto a [11 $\overline{1}]$-oriented $\mathrm{NiO}$ crystal under normal incidence, with the magnetic field pointing in the $[3,7,10]$ direction (Fig. 1). The $8 \mathrm{fs}$ pulses propagate collinearly with the terahertz field through $\mathrm{NiO}$ to monitor the Faraday rotation $\theta_{\mathrm{F}}(t)$. Unlike terahertz electromagnetic radiation reemitted by the induced magnetization $\mathbf{M}(t)$ (ref. 20), the Faraday effect scales linearly with $\mathbf{M}(t)$. The diameter of the near-infrared pulse $(15 \mu \mathrm{m})$ is chosen to be much smaller than the terahertz spot $(\sim 500 \mu \mathrm{m})$ to ensure homogeneous terahertz fields throughout the probed area

\section{References}

1. Ferguson, B. \& Zhang, X.-C. Materials for terahertz science and technology. Nature Mater. 1, 26-33 (2002).

2. Tonouchi, M. Cutting-edge terahertz technology. Nature Photon. 1 , 97-105 (2007) and references therein.

3. Huber, R. et al. How many-particle interactions develop after ultrafast excitation of an electron-hole plasma. Nature 414, 286-289 (2001).

4. Kaindl, R. A., Carnahan, M. A., Hägele, D., Lövenich, R. \& Chemla, D. S. Ultrafast terahertz probes of transient conducting and insulating phases in an electron-hole gas. Nature 423, 734-738 (2003).

5. Kröll, J. et al. Phase-resolved measurements of stimulated emission in a laser. Nature 449, 698-701 (2007).

6. Cole, B. E., Williams, J. B., King, B. T., Sherwin, M. S. \& Stanley, C. R. Coheren manipulation of semiconductor quantum bits with terahertz radiation. Nature 410, 60-63 (2001)

7. Carter, S. G. et al. Quantum coherence in an optical modulator. Science 310 , 651-653 (2005).

8. Danielson, J. R. et al. Interaction of strong single-cycle terahertz pulses with semiconductor quantum wells. Phys. Rev. Lett. 99, 237401 (2007).

9. Leinß, S. et al. Terahertz coherent control of optically dark paraexcitons in $\mathrm{Cu}_{2} \mathrm{O}$ Phys. Rev. Lett. 101, 246401 (2008).

10. Yen, T. J. et al. Terahertz magnetic response from artificial materials. Science 303, 1494-1496 (2004).

11. Linden, S. et al. Magnetic response of metamaterials at 100 terahertz. Science 306, 1351-1353 (2004)

12. Pimenov, A. et al. Magnetic and magnetoelectric excitations in $\mathrm{TbMnO}_{3}$. Phys. Rev. Lett. 102, 107203 (2009)

13. Bourges, P. et al. The spin excitation spectrum in superconducting $\mathrm{YBa}_{2} \mathrm{Cu}_{3} \mathrm{O}_{6.85}$. Science 288, 1234-1237 (2000) 
14. Hiebert, W. K., Stankiewicz, A. \& Freeman, M. R. Direct observation of magnetic relaxation in a small permalloy disc by time-resolved scanning Kerr microscopy Phys. Rev. Lett. 79, 1134-1137 (1997).

15. Back, C. H. et al. Minimum field strength in precessional magnetization reversal. Science 285, 864-867 (1999).

16. Wang, Z., Pietz, M., Walowski, J., Förster, A., Lepsa, M. I. \& Münzenberg, M Spin dynamics triggered by sub-terahertz magnetic field pulses. J. Appl. Phys. 103, 123905 (2008).

17. Kimel, A. V. et al. Ultrafast non-thermal control of magnetisation by instantaneous photomagnetic pulses. Nature 435, 655-657 (2005).

18. Kimel, A. V., Kirilyuk, A., Tsvetkov, A., Pisarev, R. V. \& Rasing, Th. Laserinduced ultrafast spin reorientation in the antiferromagnet $\mathrm{TmFeO}_{3}$. Nature 429, 850-853 (2004).

19. Duong, N. P., Satoh, T. \& Fiebig, M. Ultrafast manipulation of antiferromagnetism of NiO. Phys. Rev. Lett. 93, 117402 (2004).

20. Beaurepaire, E. et al. Coherent terahertz emission from ferromagnetic film excited by femtosecond laser pulses. Appl. Phys. Lett. 84, 3465-3467 (2004).

21. Hilton, D. J. et al. Terahertz emission via ultrashort-pulse excitation of magnetic metal films. Opt. Lett. 29, 1805-1807 (2004).

22. Blanchard, F. et al. Generation of $1.5 \mu \mathrm{J}$ single-cycle terahertz pulses by optical rectification from a large aperture $\mathrm{ZnTe}$ crystal. Opt. Express 15, 13212-13220 (2007).

23. Yeh, K.-L., Hoffmann, M. C., Hebling, J. \& Nelson, K. A. Generation of $10 \mu \mathrm{J}$ ultrashort terahertz pulses by optical rectification. Appl. Phys. Lett. 90 171121 (2007).

24. Hellwege, K. H. \& Madelung, O. (eds) Landolt-Börnstein: Numerical Data and Functional Relationships, New Series, Group III, Vol, 17g (Springer-Verlag, 1984) and references therein.

25. Sänger, I., Pavlov, V. V., Bayer, M. \& Fiebig, M. Distribution of antiferromagnetic spin and twin domains in NiO. Phys. Rev. B 74, 144401 (2006).
26. Hutchings, M. T. \& Samuelson, E. J. Measurement of spin-wave dispersion in $\mathrm{NiO}$ by inelastic neutron scattering and its relation to magnetic properties. Phys. Rev. B 6, 3447-3461 (1972).

27. Eerenstein, W., Mathur, N. D. \& Scott, J. F. Multiferroic and magnetoelectric materials. Nature 442, 759-765 (2006).

28. Stöhr, J. \& Siegmann, H. C. Magnetism: From Fundamentals to Nanoscale Dynamics (Springer-Verlag, 2006) and references therein.

29. Takahashi, S. et al. Coherent manipulation and decoherence of $S=10$ single-molecule magnets. Phys. Rev. Lett. 102, 087603 (2009).

30. Sell, A., Leitenstorfer, A. \& Huber, R. Phase-locked generation and field-resolved detection of widely tunable terahertz pulses with amplitudes exceeding $100 \mathrm{MV} / \mathrm{cm}$. Opt. Lett. 33, 2767-2769 (2008).

\section{Acknowledgements}

The authors thank L. Kuipers and U. Novak for helpful discussions. Support from the German Research Foundation (DFG) via Emmy Noether grant HU1598/1-1 and SFB767 is gratefully acknowledged.

\section{Author contributions}

T.K., A.S., R.H. and A.L. designed the experiment. Measurements were performed by T.K., A.S., R.H. and A.P. A.S., T.K. and R.H. analysed and modelled the data. M.F. prepared the sample, which was characterized by G.K., S.M. and T.D. T.K., R.H., A.S., A.L., M.W. and M.F. co-wrote the paper. All authors contributed to discussions. 